Journal of Agricultural Sciences
(Tarim Bilimleri Dergisi)

\title{
Effects of Scalding Parameters and Ripening on the Chemical, Textural and Microstructural Properties of Urfa Cheese
}

\author{
Ahmet Ferit ATASOYa* ${ }^{a}$, Mehmet ÇiÇEK ${ }^{a} \mathbb{D}$, Huriye Gözde CEYLAN ${ }^{b} \mathbb{D}$, Ali Adnan HAYALOGLU ${ }^{c} \mathbb{D}$ \\ ${ }^{a_{F a c u l t y}}$ of Engineering, Department of Food Engineering, Harran University, 63010 Sanlurfa, TURKEY

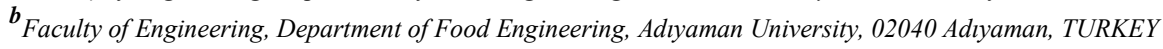

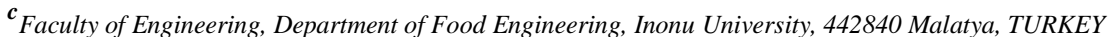

ARTICLE INFO

Research Article

Corresponding Author: Ahmet Ferit ATASOY, E-mail: fatasoy@ harran.edu.tr

Received: 10 August 2019 / Revised: 06 November 2019 / Accepted: 16 December 2019 / Online: 31 May 2021

\section{ABSTRACT}

The objectives of this study were to determine the effect of cheese scalding temperature, scalding time, and ripening time on the chemical, textural and microstructural properties of ovine milk Urfa cheese. Dry salted fresh cheeses were scalded in whey at $70{ }^{\circ} \mathrm{C}, 80^{\circ} \mathrm{C}$, and $90{ }^{\circ} \mathrm{C}$ for 5 and 10 minutes and ripened for 90 days, respectively. Scalding temperature significantly affected fat in dry matter and salt in dry matter $(\mathrm{P}<0.05)$, total solids and nitrogen, hardness, cohesiveness, gumminess, and chewiness $(\mathrm{P}<0.001)$. Scalding time significantly affected total nitrogen and gumminess $(\mathrm{P}<0.05)$, total solids, and hardness $(\mathrm{P}<0.001)$
Ripening time significantly affected chemical, textural and color properties of ovine milk Urfa cheese $(\mathrm{P}<0.001)$, except fat in dry matter $(\mathrm{P}<0.05)$. The color properties of Urfa cheese were not significantly affected by the scalding temperature and time $(\mathrm{P}>0.05)$. Besides, scalding treatments have improved the microstructure of ovine milk Urfa cheeses. Urfa cheese exhibited a more compact, coarser, and uniform structure with increasing scalding temperature and time. As a result, scalding treatments and ripening on the chemical, textural and microstructural characteristics of Urfa cheeses were substantially effective.

Keywords: Urfa cheese; Scalding; Ripening; Microstructure; Texture

(C) Ankara University, Faculty of Agriculture

\section{Introduction}

Textural features are an expression of the rheological properties of the physical structure of food. Cheese quality and identity are largely dependent on textural properties. Cheese texture is very important on consumer acceptability and the end-use of the cheese (Hort \& Le Grys 2001; Alinovi et al. 2018). Furthermore, there is a relationship between the microstructure and rheological properties of the cheese, and both are influenced by the chemical cheese composition (El Bakry \& Sheehan 2014). On the other hand, numerous reactions take place during the ripening of cheese. It is known that the texture of the cheese is extensively affected by the chemical composition of cheese, cheese production, and biochemical changes during the ripening (Tomaszewska et al. 2019).

Urfa cheese is a traditional semi-hard brined Turkish cheese variety, which is manufactured mainly in the southeast Anatolia region of Turkey from raw bovine milk or mixtures of ovine and caprine milk. However, the industrial Urfa cheese has been made from cow's milk, because of the very short lactation period of ewe's and goat's milk in Turkey. Urfa cheese is consumed fresh and/or mature. The unripened Urfa cheese is only used for the production of traditional cheese dessert products, such as "cheese helva", "cheese bread", and "kadayif" (Atasoy et al. 2013). Urfa cheese is produced without milk pasteurization and starter bacteria, and cheese microflora consists of indigenous microorganisms obtained from raw milk or transmitted from the environment. The presence of natural microorganisms provides the cheese with unique characteristics and cheeses made from raw milk are preferred by consumers (Atasoy et al. 2008; Kirmac1 2016). The microbial safety of Urfa cheese is supplied by dry salting technology by the manual spreading of salt onto the cheese surface. Alternatively, scalding of fresh cheese blocks in boiling whey is an alternative practical way of reducing microbial counts in Urfa cheese. Although there is no standard scalding temperature and time in the traditional Urfa cheese production, general scalding temperature and time are applied as $65-90{ }^{\circ} \mathrm{C}$ and about 5-10 minutes, respectively. These methods have different effects on natural microflora and also chemical, textural, and microstructural properties during storage. Following scalding and/or dry salting, Urfa cheese is ripened in brine.

Although numerous studies on the microstructural and textural characteristics of many cheese varieties were conducted, studies on the microstructural and textural characteristics of Urfa cheese are limited (Özer et al. 2003). As far as we know, no study has been conducted on the color characteristics of Urfa cheese up to now. Moreover, any study investigated the effect of 
scalding time on the properties of Urfa cheese was not encountered. The objective of this study was to investigate the effects of scalding temperature, scalding time, and ripening time on the color, chemical, textural and microstructural properties of Urfa cheese made from raw ewe milk.

\section{Material and Methods}

\subsection{Materials}

Ewe milk used in cheese production was obtained from Şanlıurfa province. The commercial rennet (Surer, Konya, Turkey) was used to coagulate the milk.

\subsection{Cheesemaking and sampling}

Fresh dry salted and scalded cheeses were designated as A5 (scalded at $70{ }^{\circ} \mathrm{C}$ for $5 \mathrm{~min}$ ), A10 (scalded at $70{ }^{\circ} \mathrm{C}$ for 10 min), B5 (scalded at $80{ }^{\circ} \mathrm{C}$ for $5 \mathrm{~min}$ ), B10 (scalded at $80^{\circ} \mathrm{C}$ for $10 \mathrm{~min}$ ), $\mathrm{C} 5$ (scalded at $90^{\circ} \mathrm{C}$ for $5 \mathrm{~min}$ ) and $\mathrm{C} 10$ (scalded at $90{ }^{\circ} \mathrm{C}$ for $10 \mathrm{~min}$ ). Cheese production is carried out according to Atasoy et al. (2013) by the traditional method. The flow diagram of experimental Urfa cheeses is demonstrated in Figure 1. The Urfa cheese production was replicated 2 times on different days. From each batch, 1, 30, 60, and 90-day old cheese were sampled and analyzed. Each batch consisted of four cheese blocks (each block contained approximately $250 \mathrm{~g}$ of cheese).

Figure 1- Flow chart of traditional Urfa cheese production

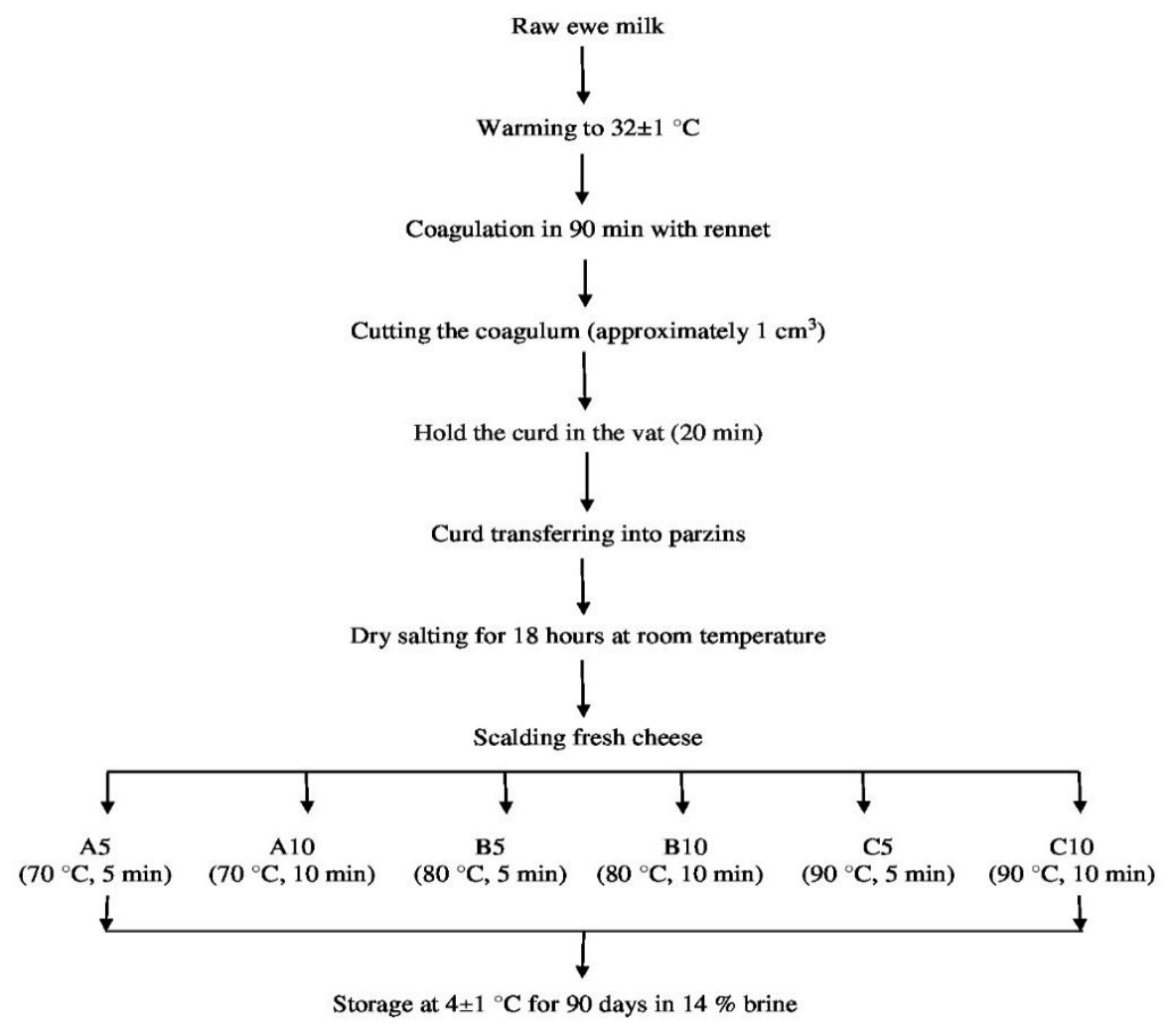

\subsection{Cheese analyses}

The titratable acidity, total solids, and salt analyses were carried out according to Turkish Standards (1989). The IDF (1993) method for total nitrogen was used. $\mathrm{pH}$ was measured by $\mathrm{pH}$ meter (Hanna, HI 2215). The fat analysis was performed with the Gerber method according to Turkish Standards (TS 1978).

Texture profile analyses were performed using Texture Analyzer Model LF Plus (Lloyd Instruments Ltd., Hampshire, UK). Color values were determined using the Hunter Lab instrument (color Quest XE, UK). Lightness, redness, yellowness values of samples were measured according to the CIE system and the $\Delta \mathrm{E}$ value of samples was calculated.

Microstructures of samples were determined as described by Hayaloglu et al. (2014). Images were monitored with a scanning electron microscope (LEO, EVO 40 Model, Carl Zeiss SMT, Oberkochen, Germany) at $20 \mathrm{kV}$. 


\subsection{Statistical analyses}

Results were performed by analysis of variance using the Minitab version 16 packet statistic programs. The differences between the samples were determined by Tukey's test.

\section{Results and Discussion}

\subsection{Chemical composition}

The effects of scalding parameters and ripening time on the chemical compositions of Urfa cheese are presented in Table 1 and the chemical compositions of the Urfa cheese samples during ripening are demonstrated in Table 2. Total solid contents of the samples were significantly $(\mathrm{P}<0.001)$ affected by scalding temperature, scalding time, and ripening time. Also, the effects of scalding temperature $(\mathrm{P}<0.001)$, scalding time $(\mathrm{P}<0.05)$ on total nitrogen contents were found to be significant. Also, the total solid and total nitrogen contents of Urfa cheese were significantly increased with increasing scalding temperature and time. This is likely to be due to whey expulsion during scalding treatments. Kahyaoglu \& Kaya (2003) stated that heat treatment applied to curds reduced the moisture content of Gaziantep cheese. Similar results have been reported by Tunick et al. (1993) for Mozzarella cheeses. Moreover, total solids and total nitrogen contents of Urfa cheese were significantly decreased during ripening. Changes in total solids can be attributed to the breakdown of peptide bonds and new ionic groups. These decreases may be due to the transition of the soluble nitrogen fractions into the brine during cheese storage. Further losses are referred to as hydrolysis of caseins by proteolytic enzymes to lower molecular weight compounds (Grappin \& Beuvier 1997) and scattering of water-soluble nitrogen into the brine (Prasad \& Alvarez 1999). Previous studies (Atasoy \& Türkoğlu 2008) showed that total nitrogen and solid contents of Urfa cheese declined during storage.

While the effect of ripening time on $\mathrm{pH}$ and titratable acidity of Urfa cheeses were found to be significant $(\mathrm{P}<0.001)$, the effects of scalding parameters were insignificant $(\mathrm{P}>0.05)$. Also, titratable acidity and $\mathrm{pH}$ values of Urfa cheese decreased and increased $(\mathrm{P}<0.05)$, respectively, until the $30^{\text {th }}$ day of ripening. However, no any change was found during the rest of the storage period. These can be explained by the continued fermentation during the maturation, completed by the end of the first month. Additionally, the decrease in titratable acidity may be due to the assimilation of lactic acid and the production of ammonia (Kırmacı et al. 2014). These results are in agreement with those of Atasoy \& Türkoğlu (2008) for Urfa cheese.

Fat in dry matter contents of Urfa cheese was significantly affected by scalding temperature, and ripening time $(\mathrm{P}<0.05)$. In particular, the C5 sample was found to have lower fat content than others. A group of researchers (Ozer et al. 2004) have reported that scalded Urfa cheeses contained higher fat than unscalded ones. Furthermore, fat contents (as dry matter) of B10, C5, and $\mathrm{C} 10$ samples decreased $(\mathrm{P}<0.05)$ during the first 30 days of ripening and remained almost constant after this day. Sahingil et al. (2014) reported that fat contents in dry matter of white cheeses depended on the ripening time.

Table 1- The variance analysis results on the effect of scalding temperature, scalding time, and ripening on the chemical composition, $L, a, b, \Delta E$ values, and textural properties of Urfa cheese samples (F-values of independent variables and interactions)

\begin{tabular}{|c|c|c|c|c|c|c|c|}
\hline & \multicolumn{7}{|c|}{ Sources of variance } \\
\hline & STE & $S T I$ & $R$ & $S T E \times S T I$ & $S T E \times R$ & $S T I \times R$ & STE $x$ STI $x R$ \\
\hline \multicolumn{8}{|c|}{ Chemical composition } \\
\hline Total solids & $64.87^{* * *}$ & $54.88^{* * *}$ & $393.10^{* * *}$ & 0.06 & 1.17 & 0.11 & 0.57 \\
\hline $\mathrm{pH}$ & 0.65 & 0.00 & $4.61^{* * * *}$ & 0.07 & 0.19 & 0.11 & 0.22 \\
\hline Titratable acidity & 0.66 & 2.22 & $85.59^{* * * *}$ & 0.92 & 0.22 & 0.58 & 1.38 \\
\hline Total nitrogen & $98.92^{* * *}$ & $7.82^{*}$ & $1283.12^{* * * *}$ & 1.50 & 2.06 & 2.38 & 1.10 \\
\hline Fat in dry matter & $5.28^{*}$ & 0.13 & $3.38^{*}$ & 1.01 & 0.94 & 0.59 & 0.36 \\
\hline Salt in dry matter & $3.96^{*}$ & 0.56 & $785.56^{* * *}$ & 0.21 & $4.35^{* *}$ & $3.53^{*}$ & 1.29 \\
\hline \multicolumn{8}{|l|}{ Texture } \\
\hline Hardness & $84.21^{* * *}$ & $19.29^{* * *}$ & $1219.70^{* * *}$ & 1.18 & $4.06^{* * *}$ & 0.43 & 0.31 \\
\hline Cohesiveness & $54.58^{* * *}$ & 0.38 & $127.23^{* * *}$ & 1.31 & $8.17^{* * *}$ & 0.13 & 1.14 \\
\hline Springiness & 0.71 & 0.55 & $135.67^{* * *}$ & 1.37 & 1.42 & 0.32 & 0.72 \\
\hline Gumminess & $112.93^{* * *}$ & $5.43^{*}$ & $574.40^{* * *}$ & 1.15 & $25.12^{* * *}$ & 0.38 & 1.10 \\
\hline Chewiness & $78.55^{* * *}$ & 4.10 & $548.53^{* * *}$ & 1.32 & $24.00^{* * * *}$ & 0.45 & 1.09 \\
\hline Fracturability & 0.06 & 0.34 & $29.02^{* * * *}$ & 0.09 & 0.98 & 0.08 & 1.08 \\
\hline Adhesiveness & $4.65^{*}$ & 1.34 & $9.78^{* * *}$ & 2.75 & $2.84^{*}$ & $3.87^{*}$ & 0.52 \\
\hline \multicolumn{8}{|l|}{ Color } \\
\hline$L$ & 0.16 & 0.16 & $15.52^{* * *}$ & 0.53 & 0.26 & 0.12 & 0.42 \\
\hline$a$ & 2.75 & 1.99 & $31.09^{* * *}$ & 1.53 & 0.23 & 0.79 & 0.61 \\
\hline$b$ & 1.89 & 1.51 & $69.24^{* * *}$ & 1.26 & 1.55 & 0.27 & 1.26 \\
\hline$\Delta \mathrm{E}$ & 0.47 & 0.35 & $6.02^{* * * *}$ & 1.27 & 1.02 & 0.56 & 0.65 \\
\hline
\end{tabular}

${ }^{*}, \mathrm{P}<0.05$ significance level; ${ }^{* *}, \mathrm{P}<0.01$ significance level; ${ }^{* * *}, \mathrm{P}<0.001$ significance level; STE, Scalding temperature; STI, Scalding time; R, Ripening time 
Salt in the dry matter contents was significantly affected by scalding temperature $(\mathrm{P}<0.05)$, ripening time $(\mathrm{P}<0.001)$, the interaction of the scalding temperature and ripening time $(\mathrm{P}<0.01)$, and scalding time and ripening time $(\mathrm{P}<0.05)$. Salt contents in the dry matter of samples increased $(\mathrm{P}<0.05)$ during the ripening. In particular, the salt contents in the dry matter of the cheese samples increased until the $60^{\text {th }}$ day of ripening then almost unchanged (except for the A5 sample). The changes in salt contents of Urfa cheeses were similar in previous studies (Atasoy et al. 2008). When cheese is placed in brine, salt molecules transfer from the brine into the cheese as a result of the osmotic pressure difference between the cheese and the brine. Until the osmotic balance between cheese and brine is reached, salt molecules diffusion continues. Thus, salt diffusion was faster into the cheese from brine at the beginning of the ripening.

Table 2- The chemical composition of Urfa cheese samples during the ripening

\begin{tabular}{|c|c|c|c|c|c|}
\hline \multirow{2}{*}{\multicolumn{2}{|c|}{ Cheese samples }} & \multicolumn{4}{|c|}{ Ripening Time (days) } \\
\hline & & 1 & 30 & 60 & 90 \\
\hline \multirow{6}{*}{$\begin{array}{l}\text { Total solids } \\
\left(\mathrm{g} 100 \mathrm{~g}^{-1}\right)\end{array}$} & A5 & $58.73 \pm 0.03^{\mathrm{Ad}}$ & $56.80 \pm 0.64^{\mathrm{ABb}}$ & $54.92 \pm 0.26^{\mathrm{Bc}}$ & $52.92 \pm 0.44^{\mathrm{Ca}}$ \\
\hline & A10 & $59.61 \pm 0.15^{\mathrm{Ac}}$ & $58.03 \pm 0.28^{\mathrm{Aab}}$ & $55.22 \pm 0.06^{\mathrm{Bbc}}$ & $53.56 \pm 0.76^{\mathrm{Ba}}$ \\
\hline & B5 & $60.71 \pm 0.09^{\mathrm{Ab}}$ & $58.09 \pm 0.29^{\mathrm{ABab}}$ & $55.86 \pm 0.60^{\mathrm{BCabc}}$ & $53.88 \pm 0.77^{\mathrm{Ca}}$ \\
\hline & B10 & $61.49 \pm 0.19^{\mathrm{Aab}}$ & $58.63 \pm 0.28^{\mathrm{Bab}}$ & $56.74 \pm 0.01^{\mathrm{Cabc}}$ & $54.29 \pm 0.02^{\mathrm{Da}}$ \\
\hline & $\mathrm{C} 5$ & $61.57 \pm 0.26^{\mathrm{Aab}}$ & $58.68 \pm 0.31^{\mathrm{Bab}}$ & $56.43 \pm 0.03^{\mathrm{Cab}}$ & $54.61 \pm 0.31^{\mathrm{Da}}$ \\
\hline & $\mathrm{C} 10$ & $61.85 \pm 0.05^{\mathrm{Aa}}$ & $59.41 \pm 0.01^{\mathrm{Ba}}$ & $57.42 \pm 0.29^{\mathrm{Ca}}$ & $55.47 \pm 0.08^{\mathrm{Da}}$ \\
\hline \multirow{6}{*}{$\mathrm{pH}$} & A5 & $5.25 \pm 0.00^{\mathrm{Ba}}$ & $5.42 \pm 0.03^{\mathrm{Aa}}$ & $5.43 \pm 0.22^{\mathrm{Aa}}$ & $5.47 \pm 0.01^{\mathrm{Aa}}$ \\
\hline & A10 & $5.26 \pm 0.10^{\mathrm{Ba}}$ & $5.32 \pm 0.07^{\mathrm{Aba}}$ & $5.46 \pm 0.12^{\mathrm{Aa}}$ & $5.49 \pm 0.09^{\mathrm{Aa}}$ \\
\hline & B5 & $5.21 \pm 0.11^{\mathrm{Ba}}$ & $5.44 \pm 0.01^{\mathrm{Aa}}$ & $5.42 \pm 0.14^{\mathrm{Aa}}$ & $5.52 \pm 0.04^{\mathrm{Aa}}$ \\
\hline & B10 & $5.23 \pm 0.07^{\mathrm{Ba}}$ & $5.34 \pm 0.15^{\mathrm{ABa}}$ & $5.40 \pm 0.01^{\mathrm{Aa}}$ & $5.58 \pm 0.16^{\mathrm{Aa}}$ \\
\hline & $\mathrm{C} 5$ & $5.15 \pm 0.12^{\mathrm{Ba}}$ & $5.31 \pm 0.09^{\mathrm{ABa}}$ & $5.42 \pm 0.16^{\mathrm{Aa}}$ & $5.40 \pm 0.18^{\mathrm{Aa}}$ \\
\hline & $\mathrm{C} 10$ & $5.24 \pm 0.00^{\mathrm{Aa}}$ & $5.42 \pm 0.20^{\mathrm{Aa}}$ & $5.35 \pm 0.18^{\mathrm{Aa}}$ & $5.38 \pm 0.18^{\mathrm{Aa}}$ \\
\hline \multirow{6}{*}{$\begin{array}{l}\text { Titratable acidity } \\
\qquad\left(\mathrm{g} 100 \mathrm{~g}^{-1} \mathrm{la}\right)\end{array}$} & A5 & $0.20 \pm 0.01^{\mathrm{Aa}}$ & $0.10 \pm 0.01^{\mathrm{Ba}}$ & $0.09 \pm 0.01^{\mathrm{Ba}}$ & $0.09 \pm 0.01^{\mathrm{Ba}}$ \\
\hline & A10 & $0.21 \pm 0.02^{\mathrm{Aa}}$ & $0.09 \pm 0.00^{\mathrm{Ba}}$ & $0.11 \pm 0.01^{\mathrm{ABa}}$ & $0.08 \pm 0.01^{\mathrm{Ba}}$ \\
\hline & B5 & $0.20 \pm 0.01^{\mathrm{Aa}}$ & $0.07 \pm 0.01^{\mathrm{Ba}}$ & $0.11 \pm 0.02^{\mathrm{ABa}}$ & $0.08 \pm 0.01 \mathrm{ABa}$ \\
\hline & B10 & $0.21 \pm 0.02^{\mathrm{Aa}}$ & $0.08 \pm 0.01^{\mathrm{Ba}}$ & $0.08 \pm 0.01^{\mathrm{Ba}}$ & $0.06 \pm 0.01^{\mathrm{Ba}}$ \\
\hline & $\mathrm{C} 5$ & $0.21 \pm 0.01^{\mathrm{Aa}}$ & $0.12 \pm 0.02^{\mathrm{ABa}}$ & $0.08 \pm 0.01^{\mathrm{Ba}}$ & $0.07 \pm 0.01^{\mathrm{Ba}}$ \\
\hline & $\mathrm{C} 10$ & $0.19 \pm 0.01^{\mathrm{Aa}}$ & $0.05 \pm 0.01^{\mathrm{Ba}}$ & $0.09 \pm 0.02^{\mathrm{Ba}}$ & $0.06 \pm 0.01^{\mathrm{Ba}}$ \\
\hline \multirow{6}{*}{$\begin{array}{l}\text { Total nitrogen } \\
\quad\left(\mathrm{g} 100 \mathrm{~g}^{-1}\right)\end{array}$} & A5 & $22.81 \pm 0.19^{\text {Acd }}$ & $19.51 \pm 0.48^{\mathrm{Bb}}$ & $17.24 \pm 0.24^{\mathrm{Cb}}$ & $15.75 \pm 0.02^{\mathrm{Cc}}$ \\
\hline & A10 & $22.61 \pm 0.15^{\text {Acd }}$ & $19.98 \pm 0.01^{\mathrm{Ba}}$ & $17.98 \pm 0.01^{\mathrm{Cab}}$ & $16.31 \pm 0.01^{\mathrm{Dbc}}$ \\
\hline & B5 & $23.20 \pm 0.07^{\mathrm{Abc}}$ & $20.21 \pm 0.03^{\mathrm{Bab}}$ & $18.18 \pm 0.31^{\mathrm{Cab}}$ & $16.88 \pm 0.17^{\mathrm{Dbc}}$ \\
\hline & B10 & $23.42 \pm 0.02^{\mathrm{Abc}}$ & $20.12 \pm 0.34^{\mathrm{Bab}}$ & $18.28 \pm 0.00^{\mathrm{Cab}}$ & $16.95 \pm 0.02^{\mathrm{Db}}$ \\
\hline & $\mathrm{C} 5$ & $24.29 \pm 0.18^{\mathrm{Aa}}$ & $20.69 \pm 0.06^{\mathrm{Bab}}$ & $18.36 \pm 0.39^{\mathrm{Cab}}$ & $17.76 \pm 0.14^{\mathrm{Ca}}$ \\
\hline & $\mathrm{C} 10$ & $23.97 \pm 0.12^{\mathrm{Aba}}$ & $20.96 \pm 0.12^{\mathrm{Ba}}$ & $18.96 \pm 0.04^{\mathrm{Ca}}$ & $18.01 \pm 0.24^{\mathrm{Da}}$ \\
\hline \multirow{6}{*}{$\begin{array}{l}\text { Fat in dry matter } \\
\qquad\left(\mathrm{g} 100 \mathrm{~g}^{-1}\right)\end{array}$} & A5 & $45.44 \pm 0.40^{\mathrm{Aa}}$ & $45.51 \pm 0.56^{\mathrm{Aa}}$ & $46.09 \pm 0.12^{\mathrm{Aa}}$ & $45.81 \pm 0.33^{\mathrm{Aa}}$ \\
\hline & A10 & $45.18 \pm 0.42^{\mathrm{Aa}}$ & $45.02 \pm 0.21^{\mathrm{Aa}}$ & $45.67 \pm 0.33^{\mathrm{Aa}}$ & $45.97 \pm 0.28^{\mathrm{Aa}}$ \\
\hline & B5 & $45.86 \pm 0.02^{\mathrm{Aa}}$ & $45.13 \pm 0.39^{\mathrm{Aa}}$ & $45.48 \pm 0.55^{\mathrm{Aa}}$ & $45.39 \pm 0.56^{\mathrm{Aa}}$ \\
\hline & B 10 & $45.48 \pm 0.19^{\mathrm{Aa}}$ & $44.76 \pm 0.21^{\mathrm{Ba}}$ & $45.27 \pm 0.56^{\mathrm{Aa}}$ & $45.71 \pm 0.14^{\mathrm{Aa}}$ \\
\hline & $\mathrm{C} 5$ & $44.99 \pm 0.08^{\mathrm{Ab}}$ & $44.46 \pm 0.40^{\mathrm{Bb}}$ & $45.07 \pm 0.57^{\mathrm{Aa}}$ & $44.91 \pm 0.37^{\mathrm{Ab}}$ \\
\hline & $\mathrm{C} 10$ & $45.87 \pm 0.26^{\mathrm{Aa}}$ & $44.50 \pm 0.32^{\mathrm{Bb}}$ & $45.01 \pm 0.06^{\mathrm{Aa}}$ & $45.06 \pm 0.60^{\mathrm{Aa}}$ \\
\hline \multirow{6}{*}{$\begin{array}{l}\text { Salt in dry matter } \\
\qquad\left(\mathrm{g} 100 \mathrm{~g}^{-1}\right)\end{array}$} & A5 & $14.63 \pm 0.06^{\mathrm{Da}}$ & $18.75 \pm 0.20^{\mathrm{Ca}}$ & $21.38 \pm 0.57^{\mathrm{Ba}}$ & $23.48 \pm 0.16^{\mathrm{Aa}}$ \\
\hline & A10 & $15.66 \pm 0.54^{\mathrm{Ca}}$ & $19.29 \pm 0.15^{\mathrm{Ba}}$ & $20.99 \pm 0.59^{\mathrm{ABa}}$ & $22.55 \pm 0.11^{\mathrm{Aab}}$ \\
\hline & B5 & $14.94 \pm 0.24^{\mathrm{Ca}}$ & $19.48 \pm 0.37^{\mathrm{Ba}}$ & $21.49 \pm 0.19^{\mathrm{Aa}}$ & $22.39 \pm 0.06^{\mathrm{Ab}}$ \\
\hline & B 10 & $14.94 \pm 0.28^{\mathrm{Ca}}$ & $20.30 \pm 0.44^{\mathrm{Ba}}$ & $21.71 \pm 0.25^{\mathrm{ABa}}$ & $22.15 \pm 0.21^{\mathrm{Ab}}$ \\
\hline & $\mathrm{C} 5$ & $14.44 \pm 0.27^{\mathrm{Ca}}$ & $19.33 \pm 0.31^{\mathrm{Ba}}$ & $21.50 \pm 0.16^{\mathrm{Aa}}$ & $21.85 \pm 0.27^{\mathrm{Ab}}$ \\
\hline & $\mathrm{C} 10$ & $14.51 \pm 0.07^{\mathrm{Ca}}$ & $19.63 \pm 0.03^{\mathrm{Ba}}$ & $21.27 \pm 0.23^{\mathrm{Aa}}$ & $21.76 \pm 0.01^{\mathrm{Ab}}$ \\
\hline
\end{tabular}

\footnotetext{
${ }^{\text {A-D }}$ Means in each row with different uppercase letters were significantly affected by storage periods $(\mathrm{P}<0.05)$. ${ }^{\mathrm{a}-\mathrm{d}} \mathrm{Means}$ with different lowercase letters were significantly different among cheese samples within the column of the similar ripening period ( $\mathrm{P}<0.05$ ). A5 (scalded at $70{ }^{\circ} \mathrm{C}$ for 5 min), A 10 (scalded at $70{ }^{\circ} \mathrm{C}$ for $10 \mathrm{~min}$ ), B5 (scalded at $80^{\circ} \mathrm{C}$ for $5 \mathrm{~min}$ ), B10 (scalded at $80^{\circ} \mathrm{C}$ for $10 \mathrm{~min}$ ), $\mathrm{C} 5$ (scalded at $90{ }^{\circ} \mathrm{C}$ for $5 \mathrm{~min}$ ) and $\mathrm{C} 10$ (scalded at $90{ }^{\circ} \mathrm{C}$ for $10 \mathrm{~min}$ ).
}

\subsection{Textural properties of Urfa cheeses}

The effects of scalding parameters and ripening time on the textural properties of Urfa cheese are shown in Table 1. Also, textural properties of Urfa cheese samples during the ripening period are given in Table 3. The hardness values of Urfa cheese were significantly influenced $(\mathrm{P}<0.001)$ by scalding temperature, scalding time, ripening time, and interaction of scalding temperature and ripening time. Also, the hardness of Urfa cheese was significantly increased with increasing scalding temperature and time. This is likely to be due to an increase in the protein concentration per unit area in the cheese matrix during scalding treatments. Tunick et al. (1993) reported that the hardness of Mozzarella cheese was influenced by curd scalding temperature. Similar results were determined in Gaziantep cheese (Kahyaoglu \& Kaya 2003). Besides, the hardness of Urfa cheese samples significantly decreased $(\mathrm{P}<0.05)$ during storage, and mature Urfa cheese was found less hard than fresh cheese. Reduction in hardness during storage may be due to proteolysis of the casein network. There is a positive correlation between the hardness of cheese and the quantity of intact $\alpha_{\mathrm{s} 1}$-casein (Lawrence et al. 1987). The products of proteolysis are generally water-soluble and cannot contribute 
to the protein network (Lawrence et al. 1987). The decreases in cheese during the ripening period were also observed by some researchers (Sahan et al. 2008). Reduction in the hardness of cheeses was not surprising considering the decreased total nitrogen and total solid contents (Table 2) during the ripening.

Table 3- The change of textural properties of Urfa cheese samples during the ripening

\begin{tabular}{|c|c|c|c|c|c|}
\hline & \multirow{2}{*}{ Cheese samples } & \multicolumn{4}{|c|}{ Ripening Time (days) } \\
\hline & & 1 & 30 & 60 & 90 \\
\hline \multirow{6}{*}{ Hardness } & A5 & $28.66 \pm 0.54^{\mathrm{Acd}}$ & $20.02 \pm 0.96^{\mathrm{Bcd}}$ & $10.25 \pm 0.73^{\mathrm{Cb}}$ & $6.22 \pm 0.39^{\mathrm{Db}}$ \\
\hline & A10 & $31.63 \pm 1.31^{\mathrm{Abc}}$ & $22.44 \pm 0.37^{\mathrm{Bbcd}}$ & $12.77 \pm 0.04^{\mathrm{Cab}}$ & $6.81 \pm 0.50^{\mathrm{Dab}}$ \\
\hline & B5 & $32.71 \pm 1.26^{\text {Aabcd }}$ & $24.13 \pm 0.68^{\mathrm{Babc}}$ & $14.39 \pm 0.75^{\mathrm{Cabc}}$ & $6.98 \pm 0.84^{\mathrm{Dab}}$ \\
\hline & B 10 & $33.48 \pm 1.10^{\mathrm{Aabc}}$ & $25.63 \pm 0.85^{\mathrm{Bab}}$ & $15.70 \pm 1.13^{\mathrm{Ca}}$ & $8.09 \pm 0.55^{\mathrm{Dab}}$ \\
\hline & $\mathrm{C} 5$ & $36.97 \pm 1.03^{\mathrm{Aab}}$ & $26.16 \pm 0.82^{\mathrm{Bab}}$ & $16.01 \pm 0.78^{\mathrm{Ca}}$ & $8.91 \pm 0.49^{\mathrm{Dab}}$ \\
\hline & $\mathrm{C} 10$ & $38.48 \pm 1.00^{\mathrm{Aa}}$ & $27.08 \pm 0.16^{\mathrm{Ba}}$ & $16.81 \pm 0.61^{\mathrm{Ca}}$ & $9.62 \pm 0.57^{\mathrm{Da}}$ \\
\hline \multirow{6}{*}{ Cohesiveness } & A5 & $0.16 \pm 0.01^{\mathrm{Ab}}$ & $0.14 \pm 0.01^{\mathrm{Aa}}$ & $0.13 \pm 0.01^{\mathrm{ABa}}$ & $0.08 \pm 0.00^{\mathrm{Bb}}$ \\
\hline & A10 & $0.16 \pm 0.01^{\mathrm{Ab}}$ & $0.14 \pm 0.01^{\mathrm{ABa}}$ & $0.14 \pm 0.01^{\mathrm{ABa}}$ & $0.11 \pm 0.01^{\mathrm{Bab}}$ \\
\hline & B5 & $0.26 \pm 0.01^{\mathrm{Aa}}$ & $0.18 \pm 0.01^{\mathrm{Ba}}$ & $0.17 \pm 0.01^{\mathrm{BCa}}$ & $0.11 \pm 0.01^{\mathrm{Cab}}$ \\
\hline & B10 & $0.24 \pm 0.01^{\mathrm{Aa}}$ & $0.19 \pm 0.01^{\mathrm{ABa}}$ & $0.16 \pm 0.01^{\mathrm{BCa}}$ & $0.11 \pm 0.01^{\mathrm{Cab}}$ \\
\hline & $\mathrm{C} 5$ & $0.26 \pm 0.01^{\mathrm{Aa}}$ & $0.19 \pm 0.01^{\mathrm{Ba}}$ & $0.16 \pm 0.01^{\mathrm{Ba}}$ & $0.12 \pm 0.01^{\mathrm{Ba}}$ \\
\hline & $\mathrm{C} 10$ & $0.28 \pm 0.01^{\mathrm{Aa}}$ & $0.21 \pm 0.01^{\mathrm{ABa}}$ & $0.16 \pm 0.01^{\mathrm{BCa}}$ & $0.11 \pm 0.01^{\mathrm{Cab}}$ \\
\hline \multirow{6}{*}{ Springiness } & A5 & $0.48 \pm 0.01^{\mathrm{Aa}}$ & $0.29 \pm 0.01^{\mathrm{Ba}}$ & $0.32 \pm 0.01^{\mathrm{BCa}}$ & $0.35 \pm 0.01^{\mathrm{Ca}}$ \\
\hline & A10 & $0.45 \pm 0.01^{\mathrm{Aa}}$ & $0.30 \pm 0.01^{\mathrm{Ba}}$ & $0.31 \pm 0.01^{\mathrm{Ba}}$ & $0.34 \pm 0.01^{\mathrm{Ba}}$ \\
\hline & B5 & $0.44 \pm 0.01^{\mathrm{Aa}}$ & $0.31 \pm 0.01^{\mathrm{Ba}}$ & $0.31 \pm 0.01^{\mathrm{Ba}}$ & $0.33 \pm 0.01^{\mathrm{Ba}}$ \\
\hline & B10 & $0.46 \pm 0.02^{\mathrm{Aa}}$ & $0.31 \pm 0.01^{\mathrm{Ba}}$ & $0.31 \pm 0.01^{\mathrm{Ba}}$ & $0.36 \pm 0.02^{\mathrm{Aa}}$ \\
\hline & $\mathrm{C} 5$ & $0.46 \pm 0.01^{\mathrm{Aa}}$ & $0.34 \pm 0.01^{\mathrm{Ba}}$ & $0.29 \pm 0.02^{\mathrm{Ba}}$ & $0.33 \pm 0.01^{\mathrm{Ba}}$ \\
\hline & $\mathrm{C} 10$ & $0.48 \pm 0.01^{\mathrm{Aa}}$ & $0.33 \pm 0.01^{\mathrm{Ba}}$ & $0.32 \pm 0.01^{\mathrm{Ba}}$ & $0.35 \pm 0.02^{\mathrm{Ba}}$ \\
\hline \multirow{6}{*}{ Gumminess } & A5 & $4.79 \pm 0.42^{\mathrm{Ac}}$ & $2.89 \pm 0.05^{\mathrm{Bb}}$ & $1.33 \pm 0.07^{\mathrm{Cb}}$ & $0.56 \pm 0.03^{\mathrm{Cb}}$ \\
\hline & A10 & $5.25 \pm 0.53^{\mathrm{Ac}}$ & $3.19 \pm 0.19^{\mathrm{Bb}}$ & $1.88 \pm 0.09^{\mathrm{BCab}}$ & $0.76 \pm 0.03^{\mathrm{Cab}}$ \\
\hline & B5 & $8.71 \pm 0.05^{\mathrm{Aba}}$ & $4.37 \pm 0.25^{\mathrm{Bab}}$ & $2.51 \pm 0.00^{\mathrm{Ca}}$ & $0.79 \pm 0.13^{\mathrm{Dab}}$ \\
\hline & B10 & $8.16 \pm 0.56^{\mathrm{Ab}}$ & $4.88 \pm 0.59^{\mathrm{Bab}}$ & $2.57 \pm 0.34^{\mathrm{BCa}}$ & $0.89 \pm 0.11^{\mathrm{Cab}}$ \\
\hline & $\mathrm{C} 5$ & $9.83 \pm 0.23^{\mathrm{Aab}}$ & $5.11 \pm 0.59^{\mathrm{Bab}}$ & $2.58 \pm 0.04^{\mathrm{Ca}}$ & $1.15 \pm 0.01^{\mathrm{Ca}}$ \\
\hline & $\mathrm{C} 10$ & $11.06 \pm 0.56^{\mathrm{Aa}}$ & $5.66 \pm 0.43^{\mathrm{Ba}}$ & $2.82 \pm 0.21^{\mathrm{Ca}}$ & $1.09 \pm 0.06^{\mathrm{Ca}}$ \\
\hline \multirow{6}{*}{ Chewiness } & A5 & $2.33 \pm 0.15^{\mathrm{Ac}}$ & $0.85 \pm 0.01^{\mathrm{Bb}}$ & $0.42 \pm 0.01^{\mathrm{BCb}}$ & $0.19 \pm 0.02^{\mathrm{Ca}}$ \\
\hline & A10 & $2.41 \pm 0.25^{\mathrm{Ac}}$ & $0.97 \pm 0.08^{\mathrm{Bb}}$ & $0.58 \pm 0.01^{\mathrm{Bab}}$ & $0.26 \pm 0.02^{\mathrm{Ba}}$ \\
\hline & B5 & $3.87 \pm 0.07^{\mathrm{Aabc}}$ & $1.38 \pm 0.02^{\mathrm{Bab}}$ & $0.79 \pm 0.04^{\mathrm{Cab}}$ & $0.27 \pm 0.05^{\mathrm{Da}}$ \\
\hline & B10 & $3.76 \pm 0.47^{\mathrm{Abc}}$ & $1.50 \pm 0.08^{\mathrm{Bab}}$ & $0.80 \pm 0.14^{\mathrm{Bab}}$ & $0.32 \pm 0.05^{\mathrm{Ba}}$ \\
\hline & $\mathrm{C} 5$ & $4.55 \pm 0.03^{\mathrm{Aab}}$ & $1.76 \pm 0.23^{\mathrm{Ba}}$ & $0.75 \pm 0.07^{\mathrm{Cab}}$ & $0.38 \pm 0.01^{\mathrm{Ca}}$ \\
\hline & $\mathrm{C} 10$ & $5.33 \pm 0.37^{\mathrm{Aa}}$ & $1.90 \pm 0.19^{\mathrm{Ba}}$ & $0.91 \pm 0.01^{\mathrm{BCa}}$ & $0.38 \pm 0.01^{\mathrm{Ca}}$ \\
\hline \multirow{6}{*}{ Fracturability } & A5 & $0.91 \pm 0.08^{\mathrm{Aa}}$ & $0.82 \pm 0.01^{\mathrm{Aa}}$ & $0.71 \pm 0.03^{\mathrm{Aa}}$ & $0.62 \pm 0.04^{\mathrm{Aa}}$ \\
\hline & A10 & $0.85 \pm 0.08^{\mathrm{ABa}}$ & $0.89 \pm 0.01^{\mathrm{Aa}}$ & $0.57 \pm 0.01^{\mathrm{ACa}}$ & $0.61 \pm 0.02^{\mathrm{BCa}}$ \\
\hline & B5 & $0.82 \pm 0.16^{\mathrm{Aa}}$ & $0.96 \pm 0.01^{\mathrm{Aa}}$ & $0.59 \pm 0.05^{\mathrm{Aa}}$ & $0.64 \pm 0.01^{\mathrm{Aa}}$ \\
\hline & B 10 & $0.89 \pm 0.14^{\mathrm{Aa}}$ & $0.94 \pm 0.07^{\mathrm{Aa}}$ & $0.58 \pm 0.04^{\mathrm{Aa}}$ & $0.61 \pm 0.01^{\mathrm{Aa}}$ \\
\hline & $\mathrm{C} 5$ & $0.90 \pm 0.02^{\mathrm{Aa}}$ & $0.89 \pm 0.09^{\mathrm{Aa}}$ & $0.61 \pm 0.01^{\mathrm{Aa}}$ & $0.62 \pm 0.01^{\mathrm{Aa}}$ \\
\hline & $\mathrm{C} 10$ & $0.84 \pm 0.08^{\mathrm{Aa}}$ & $0.75 \pm 0.01^{\mathrm{Aa}}$ & $0.67 \pm 0.06^{\mathrm{Aa}}$ & $0.68 \pm 0.02^{\mathrm{Aa}}$ \\
\hline \multirow{6}{*}{ Adhesiveness } & A5 & $0.09 \pm 0.01^{\mathrm{Aa}}$ & $0.04 \pm 0.01^{\mathrm{ABa}}$ & $0.01 \pm 0.01^{\mathrm{ABa}}$ & $0.03 \pm 0.02^{\mathrm{Ba}}$ \\
\hline & A10 & $0.08 \pm 0.01^{\mathrm{Aab}}$ & $0.09 \pm 0.01^{\mathrm{Aa}}$ & $0.06 \pm 0.01^{\mathrm{Aa}}$ & $0.04 \pm 0.03^{\mathrm{Aa}}$ \\
\hline & B5 & $0.10 \pm 0.01^{\mathrm{ABa}}$ & $0.07 \pm 0.01^{\mathrm{BAa}}$ & $0.04 \pm 0.01 \mathrm{ABa}$ & $0.02 \pm 0.01^{\mathrm{Ba}}$ \\
\hline & B10 & $0.06 \pm 0.01^{\mathrm{ABabc}}$ & $0.10 \pm 0.01^{\mathrm{Aa}}$ & $0.03 \pm 0.02^{\mathrm{ABa}}$ & $0.01 \pm 0.01^{\mathrm{Ba}}$ \\
\hline & $\mathrm{C} 5$ & $0.03 \pm 0.01^{\mathrm{Abc}}$ & $0.03 \pm 0.01^{\mathrm{Aa}}$ & $0.02 \pm 0.01^{\mathrm{Aa}}$ & $0.02 \pm 0.01^{\mathrm{Aa}}$ \\
\hline & $\mathrm{C} 10$ & $0.02 \pm 0.01^{\mathrm{Ac}}$ & $0.07 \pm 0.02^{\mathrm{Aa}}$ & $0.02 \pm 0.01^{\mathrm{Aa}}$ & $0.05 \pm 0.02^{\mathrm{Aa}}$ \\
\hline \multirow{6}{*}{ Stiffness } & A5 & $87.61 \pm 1.42^{\mathrm{Ab}}$ & $29.47 \pm 0.88^{\mathrm{Ba}}$ & $28.96 \pm 0.14^{\mathrm{Ba}}$ & $26.03 \pm 2.62^{\mathrm{Ba}}$ \\
\hline & A10 & $96.81 \pm 4.15^{\mathrm{Aab}}$ & $30.44 \pm 1.30^{\mathrm{Ba}}$ & $30.48 \pm 0.25^{\mathrm{Ba}}$ & $26.34 \pm 2.52^{\mathrm{Ba}}$ \\
\hline & B5 & $93.03 \pm 5.46^{\mathrm{Aab}}$ & $30.14 \pm 0.89^{\mathrm{Ba}}$ & $28.43 \pm 1.27^{\mathrm{Ba}}$ & $27.78 \pm 1.93^{\mathrm{Ba}}$ \\
\hline & B10 & $108.10 \pm 8.25^{\mathrm{Aab}}$ & $34.68 \pm 2.01^{\mathrm{Ba}}$ & $32.01 \pm 2.40^{\mathrm{Ba}}$ & $26.49 \pm 2.39^{\mathrm{Ba}}$ \\
\hline & $\mathrm{C} 5$ & $119.07 \pm 9.70^{\mathrm{Aab}}$ & $33.01 \pm 2.57^{\mathrm{Ba}}$ & $35.45 \pm 1.03^{\mathrm{Ba}}$ & $25.71 \pm 0.89^{\mathrm{Ba}}$ \\
\hline & $\mathrm{C} 10$ & $121.11 \pm 5.21^{\mathrm{Aa}}$ & $33.62 \pm 0.83^{\mathrm{Ba}}$ & $34.68 \pm 1.50^{\mathrm{Ba}}$ & $26.80 \pm 0.84^{\mathrm{Ba}}$ \\
\hline
\end{tabular}

${ }^{\text {A-D }}$ Means in each row with different uppercase letters were significantly affected by storage periods $(\mathrm{P}<0.05)$. ${ }^{\mathrm{a}-\mathrm{d}} \mathrm{Means}$ with different lowercase letters were significantly different among cheese samples within the column of the similar ripening period $(\mathrm{P}<0.05)$. A5 (scalded at $70{ }^{\circ} \mathrm{C}$ for 5 min), $\mathrm{A} 10$ (scalded at $70{ }^{\circ} \mathrm{C}$

for $10 \mathrm{~min}$ ), B5 (scalded at $80^{\circ} \mathrm{C}$ for $5 \mathrm{~min}$ ), B10 (scalded at $80^{\circ} \mathrm{C}$ for $10 \mathrm{~min}$ ), $\mathrm{C} 5$ (scalded at $90^{\circ} \mathrm{C}$ for $5 \mathrm{~min}$ ) and $\mathrm{C} 10$ (scalded at $90{ }^{\circ} \mathrm{C}$ for $10 \mathrm{~min}$ ).

The effects of scalding temperature, ripening time, and interaction of scalding temperature and ripening time on cohesiveness values of cheeses were found to be effective $(\mathrm{P}<0.001)$. Cohesiveness values of fresh cheeses generally increased with increasing scalding temperature. It has been expressed that scalded Urfa cheeses had a higher cohesiveness value than unscalded ones (Özer et al. 2003). Moreover, cohesiveness values slightly decreased $(\mathrm{P}<0.05)$ with the ripening period. These changes of cohesiveness during the ripening are in agreement with the results of some researchers (Akalın \& Karaman 2010; Eroglu et al. 2016). This may be due to proteolysis. Because proteolysis breaks the structural integrity of the protein network, leading to decreased cohesiveness (Awad et al. 2005). 
Even though springiness was affected $(\mathrm{P}<0.001)$ by the ripening time, it was not affected by the scalding parameters. However, some researchers (Hayaloglu et al. 2014) reported that the scalding process is required to provide elasticity in cheese. Özer et al. (2003) observed that scalding treatment increased the springiness value of Urfa cheeses. Also, springiness values of samples decreased $(\mathrm{P}<0.05)$ until the 30 days of ripening, then remained constant (except for A5 and B10 samples). The decreases in springiness during cheese storage may be due to degradation of the protein, especially the hydrolysis of para $\kappa$-caseinate molecules (Awad et al. 2005), and reduction of free water. Hort \& Le Grys (2001) reported that the springiness of Cheddar cheese decreased during the ripening, but also somewhat remained constant in the middle period of ripening.

The effects of ripening time, scalding temperature, the interaction of scalding temperature and ripening time $(\mathrm{P}<0.001)$, and scalding time $(\mathrm{P}<0.05)$ on the gumminess values of Urfa cheese were significantly found. This textural parameter of Urfa cheese was significantly increased with increasing scalding temperature. The variation in the aforementioned textural characteristic during the scalding was due largely to the variations in the scores for hardness. Similar results were also obtained from Kahyaoglu et al. (2005) for Gaziantep cheese which is scalded and ripened in brine like Urfa cheese. Moreover, gumminess values of Urfa cheese statistically declined $(\mathrm{P}<0.05)$ during cheese ripening. Increasing the level of $\mathrm{NaCl}$ during cheese maturation may be decreasing in the gumminess parameter due to increased water retention in the cheese. Eroglu et al. (2016) reported that the gumminess value of Kashar cheese was influenced by ripening time. Gumminess is the product of hardness and cohesiveness (Famenin et al. 2019). For this reason, the variables affecting cohesiveness and hardness also affect values of the gumminess during the ripening.

Chewiness values of Urfa cheese were influenced by the ripening time, scalding temperature, interaction of scalding temperature, and ripening time $(\mathrm{P}<0.001)$. As the scalding temperature increased, chewiness values of Urfa cheeses were generally increased. Furthermore, a decrease in chewiness values of cheeses was observed during the first 60 days of ripening $(\mathrm{P}<0.05)$. The chewiness is one of the secondary textural parameters of the cheeses and it is calculated by the hardness, cohesiveness, and springiness of the product (Famenin et al. 2019). Thus, changes in chewiness values may be associated with the variations of the hardness, cohesiveness, and springiness values during the ripening.

Fracturability was only influenced by ripening time $(\mathrm{P}<0.001)$. Nuñez et al. (1991) reported that fracturability value of ewe's milk Manchego cheese was affected by ripening time. Only fracturability value of A10 sample showed a fluctuation during the ripening $(\mathrm{P}<0.05)$. The higher fracturability value means the lower fracturability of the product (Aminifar et al. 2010). The fracturability value of the A10 sample decreased from the $30^{\text {th }}$ day of maturation, which means that the A10 sample was more fragile during the maturation. This may be related to pore formation during maturation (Aminifar \& Emam-Djomeh 2014). These findings are in good agreement with previous studies for different brined cheese (Aminifar \& Emam-Djomeh 2014).

Ripening time $(\mathrm{P}<0.001)$, scalding temperature, interactions of scalding temperature and ripening time, and scalding time and ripening time $(\mathrm{P}<0.05)$ on the adhesiveness of samples were found effective. Adhesiveness value of only A5 cheese significantly reduced $(\mathrm{P}<0.05)$ during the ripening. Sahingil et al. (2014) pointed out that the adhesiveness values of white cheeses slightly decreased during maturation, but these declines were not significant. Also, the increase in scalding temperature caused a decrease in the adhesiveness values of 1-day old cheeses. It is known that the adhesiveness value is closely related to the fat and total solid content of the cheese (Motevalizadeh et al. 2018). This change in adhesiveness was following the changes in the total solid (Table 2) and fat (data not shown) during the ripening period.

Stiffness values, in other words, firmness, were influenced by the ripening time, scalding temperature, interaction of scalding temperature and ripening time $(\mathrm{P}<0.001)$, and scalding time $(\mathrm{P}<0.05)$. Although stiffness values of samples decreased $(\mathrm{P}<0.05)$ until the $30^{\text {th }}$ day of ripening, they did not change during the rest of the maturation period. The decreases in springiness during the first month of storage may be due to increased moisture content and $\mathrm{pH}$ and breakdown of proteins (Table 2). It is known that the firmness of fresh cheeses decreases after the ripening due to biochemical and physical changes (Aminifar et al. 2010). Furthermore, there is a close relationship between the firmness of cheese and intact $\alpha_{\mathrm{s} 1}$-casein. This reduction of stiffness was not surprising due to proteolysis (Lawrence et al. 1987). Also, stiffness values of Urfa cheeses increased with the scalding treatment. This is likely to be due to whey removal and increasing total solid content of Urfa cheese. Additionally, the curd shrinks in size and becomes firmer during the scalding process. Especially, the stiffness value of fresh C10 samples was found higher than fresh A5 sample.

\subsection{Microstructures of Urfa cheeses}

Scanning electron micrographs of samples are presented in Figure 2. The microstructures of cheeses were evaluated before the ripening process. It was observed that scalding treatment was affected microstructures of Urfa cheeses. Scalding treatment resulted in a coarser and more uniform microstructure in Urfa cheeses. It has been stated that the scalding process gives a more compact structure in Malatya cheese (Hayaloglu et al. 2010). Özer et al. (2003) also reported that the scalding provided a more homogeneous microstructure in Urfa cheeses. Especially, it has been found that the cheeses have a more compact structure with increasing scalding time. Scalded cheeses for 10 minutes were characterized by a compact protein network, with small pores. The relationship between cheese microstructure and rheology is known (Buffa et al. 2001). These changes in the microstructure can be associated with textural changes. Thus, it has been determined that some of the textural properties of the Urfa cheeses are 
changed by scalding (Table 1). Akalın \& Karaman (2010) pointed out that the compactness of the protein network demonstrates the structural development of the cheese responsible for the increased hardness value.
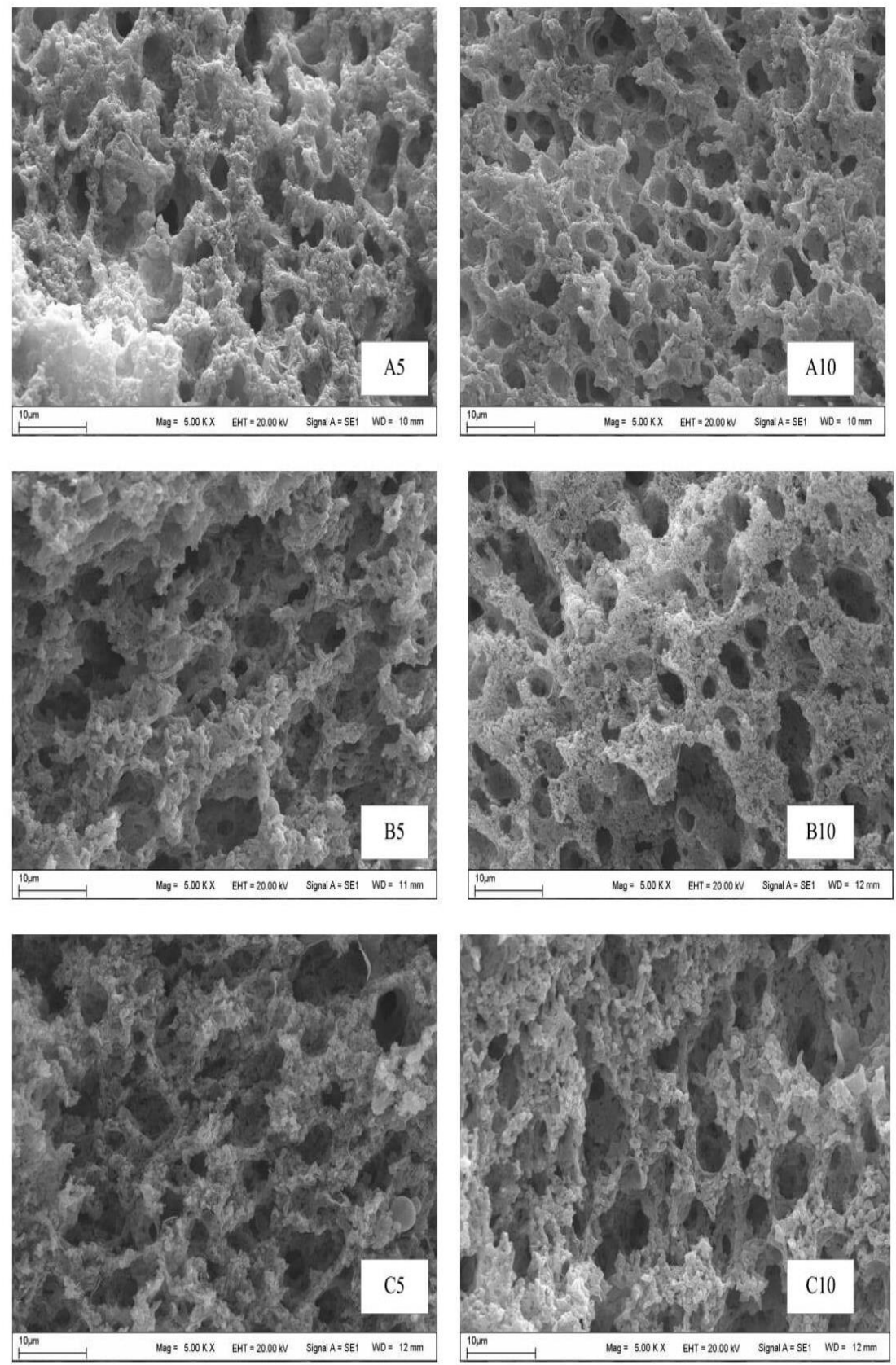

Figure 2- Scanning electron micrographs of Urfa cheese samples. Magnification is 5,000x

\subsection{Color values of Urfa cheeses}

The effects of scalding parameters and ripening time on $L, a, b$, and $\Delta \mathrm{E}$ values of samples are depicted in Table 1 . The changes in $L, a, b$ and $\Delta \mathrm{E}$ values of the Urfa cheeses during the maturation are shown in Table 4 . The color properties of Urfa cheeses were only influenced by ripening time $(\mathrm{P}<0.001)$. It was reported that the effect of ripening time on color values of cheeses was found significant by some researchers (Buffa et al. 2001). The $L, b, a$, and $\Delta \mathrm{E}$ values of cheeses did not show a definite trend throughout the ripening. The change in $L$ value of the only B10 sample was found to significant during ripening $(\mathrm{P}<0.05)$. On the other hand, $a$ values of cheeses (except for A5 and B10) were observed fluctuating $(\mathrm{P}<0.05)$ during the maturation. 90 -day old cheese generally exhibited higher $a$ value than fresh cheeses. $b$ values were changed $(\mathrm{P}<0.05)$ from $1^{\text {st }}$ day of the ripening period and they showed a higher yellowness value than 90-day old cheese. Finally, $\Delta \mathrm{E}$ values of B10 and C5 samples were found to decline $(\mathrm{P}<0.05)$ at the end of maturation. Color changes may be attributed to microstructural changes of Urfa cheeses. The scattering of light is connected to its heterogeneousness at the microstructural and molecular levels. In a firm material such as cheese, light diffuses the superficial layers and penetrates largely at the interfaces of the fat globule and the edges of whey pockets (Madadlou et al. 2006). 
Table 4- The changes in $L, a, b$, and $\Delta E$ values of Urfa cheese samples during the ripening

\begin{tabular}{|c|c|c|c|c|c|}
\hline \multirow{2}{*}{\multicolumn{2}{|c|}{ Cheese samples }} & \multicolumn{4}{|c|}{ Ripening Time (days) } \\
\hline & & 1 & 30 & 60 & 90 \\
\hline \multirow{6}{*}{$L$} & A5 & $82.52 \pm 0.01^{\mathrm{Aa}}$ & $80.32 \pm 1.67^{\mathrm{Aa}}$ & $82.67 \pm 0.51^{\mathrm{Aa}}$ & $83.10 \pm 1.99^{\mathrm{Aa}}$ \\
\hline & A10 & $85.27 \pm 1.75^{\mathrm{Aa}}$ & $82.10 \pm 0.01^{\mathrm{Aa}}$ & $82.49 \pm 0.40^{\mathrm{Aa}}$ & $82.55 \pm 0.12^{\mathrm{Aa}}$ \\
\hline & B5 & $84.79 \pm 2.47^{\mathrm{Aa}}$ & $81.40 \pm 1.12^{\mathrm{Aa}}$ & $82.63 \pm 0.84^{\mathrm{Aa}}$ & $80.68 \pm 1.05^{\mathrm{Aa}}$ \\
\hline & B10 & $86.09 \pm 1.09^{\mathrm{Aa}}$ & $81.43 \pm 0.53^{\mathrm{Ba}}$ & $82.90 \pm 0.58^{\mathrm{ABa}}$ & $82.53 \pm 0.03^{\mathrm{ABa}}$ \\
\hline & $\mathrm{C} 5$ & $85.87 \pm 1.80^{\mathrm{Aa}}$ & $82.10 \pm 2.08^{\mathrm{Aa}}$ & $82.47 \pm 0.46^{\mathrm{Aa}}$ & $81.87 \pm 0.30^{\mathrm{Aa}}$ \\
\hline & $\mathrm{C} 10$ & $85.37 \pm 0.24^{\mathrm{Aa}}$ & $81.66 \pm 1.44^{\mathrm{Aa}}$ & $82.32 \pm 0.44^{\mathrm{Aa}}$ & $81.94 \pm 0.38^{\mathrm{Aa}}$ \\
\hline \multirow{6}{*}{$a$} & A5 & $-1.41 \pm 0.07^{\mathrm{Aa}}$ & $-1.50 \pm 0.05^{\mathrm{Aa}}$ & $-1.50 \pm 0.04^{\mathrm{Ab}}$ & $-1.33 \pm 0.13^{\mathrm{Aa}}$ \\
\hline & A 10 & $-1.38 \pm 0.06^{\mathrm{Aa}}$ & $-1.53 \pm 0.01^{\mathrm{Ba}}$ & $-1.46 \pm 0.03^{\mathrm{Ba}}$ & $-1.11 \pm 0.02^{\mathrm{Ba}}$ \\
\hline & B5 & $-1.36 \pm 0.07^{\mathrm{ABa}}$ & $-1.50 \pm 0.03^{\mathrm{Ba}}$ & $-1.44 \pm 0.03^{\mathrm{ABa}}$ & $-1.22 \pm 0.02^{\mathrm{Aa}}$ \\
\hline & B10 & $-1.33 \pm 0.12^{\mathrm{Aa}}$ & $-1.45 \pm 0.03^{\mathrm{Aa}}$ & $-1.37 \pm 0.02^{\mathrm{Aa}}$ & $-1.16 \pm 0.05^{\mathrm{Aa}}$ \\
\hline & $\mathrm{C} 5$ & $-1.30 \pm 0.01^{\mathrm{ABa}}$ & $-1.48 \pm 0.04^{\mathrm{Ba}}$ & $-1.38 \pm 0.07^{\mathrm{ABa}}$ & $-1.17 \pm 0.03^{\mathrm{ABa}}$ \\
\hline & $\mathrm{C} 10$ & $-1.36 \pm 0.04^{\mathrm{ABa}}$ & $-1.50 \pm 0.01^{\mathrm{Ba}}$ & $-1.36 \pm 0.02^{\mathrm{Ba}}$ & $-1.19 \pm 0.02^{\mathrm{Aa}}$ \\
\hline \multirow{6}{*}{$b$} & A5 & $16.17 \pm 0.36^{\mathrm{Aa}}$ & $13.12 \pm 0.91^{\mathrm{ABa}}$ & $12.77 \pm 0.37^{\mathrm{Ba}}$ & $13.82 \pm 0.21^{\mathrm{ABa}}$ \\
\hline & A10 & $15.75 \pm 0.09^{\mathrm{Aa}}$ & $13.41 \pm 0.34^{\mathrm{Ba}}$ & $13.32 \pm 0.12^{\mathrm{Ba}}$ & $13.09 \pm 0.07^{\mathrm{Bab}}$ \\
\hline & B5 & $16.23 \pm 0.31^{\mathrm{Aa}}$ & $13.95 \pm 0.42^{\mathrm{Ba}}$ & $13.56 \pm 0.06^{\mathrm{Ba}}$ & $12.71 \pm 0.11^{\mathrm{Bb}}$ \\
\hline & B10 & $15.53 \pm 0.90^{\mathrm{Aa}}$ & $13.64 \pm 0.08^{\mathrm{ABa}}$ & $12.51 \pm 0.05^{\mathrm{ABa}}$ & $12.91 \pm 0.35^{\mathrm{Bab}}$ \\
\hline & $\mathrm{C} 5$ & $15.60 \pm 0.51^{\mathrm{Aa}}$ & $12.62 \pm 0.53^{\mathrm{Ba}}$ & $12.56 \pm 0.34^{\mathrm{Ba}}$ & $13.11 \pm 0.16^{\mathrm{Bab}}$ \\
\hline & $\mathrm{C} 10$ & $16.05 \pm 0.36^{\mathrm{Aa}}$ & $12.35 \pm 0.85^{\mathrm{Ba}}$ & $13.39 \pm 0.04^{\mathrm{ABa}}$ & $12.81 \pm 0.09^{\mathrm{Bab}}$ \\
\hline \multirow{6}{*}{$\Delta \mathrm{E}$} & A5 & $88.05 \pm 0.06^{\mathrm{Aa}}$ & $81.40 \pm 1.80^{\mathrm{Aa}}$ & $83.66 \pm 0.45^{\mathrm{Aa}}$ & $84.25 \pm 2.00^{\mathrm{Aa}}$ \\
\hline & A10 & $86.71 \pm 1.75^{\mathrm{Aa}}$ & $69.71 \pm 13.42^{\mathrm{Aa}}$ & $83.57 \pm 0.37^{\mathrm{Aa}}$ & $83.72 \pm 0.13^{\mathrm{Aa}}$ \\
\hline & B5 & $86.35 \pm 2.36^{\mathrm{Aa}}$ & $82.61 \pm 1.04^{\mathrm{Aa}}$ & $83.75 \pm 081^{\mathrm{Aa}}$ & $81.81 \pm 0.94^{\mathrm{Aa}}$ \\
\hline & B10 & $87.49 \pm 0.91^{\mathrm{Aa}}$ & $82.47 \pm 0.42^{\mathrm{Ba}}$ & $83.93 \pm 0.65^{\mathrm{Ba}}$ & $83.54 \pm 0.09^{\mathrm{Ba}}$ \\
\hline & $\mathrm{C} 5$ & $87.29 \pm 1.67^{\mathrm{Aa}}$ & $80.66 \pm 0.08^{\mathrm{Ba}}$ & $83.33 \pm 0.41^{\mathrm{ABa}}$ & $82.17 \pm 1.06^{\mathrm{ABa}}$ \\
\hline & $\mathrm{C} 10$ & $86.91 \pm 0.14^{\mathrm{Aa}}$ & $82.61 \pm 1.55^{\mathrm{Aa}}$ & $83.42 \pm 0.45^{\mathrm{Aa}}$ & $82.95 \pm 0.39^{\mathrm{Aa}}$ \\
\hline
\end{tabular}

${ }^{\text {A-D }}$ Means in each row with different uppercase letters were significantly affected by storage periods $(\mathrm{P}<0.05) .{ }^{\mathrm{a}-\mathrm{d}} \mathrm{Means}$ with different lowercase letters were significantly different among cheese samples within the column of the similar ripening period ( $\mathrm{P}<0.05$ ). A5 (scalded at $70{ }^{\circ} \mathrm{C}$ for 5 min), A10 (scalded at $70{ }^{\circ} \mathrm{C}$ for $10 \mathrm{~min}$ ), B5 (scalded at $80^{\circ} \mathrm{C}$ for $5 \mathrm{~min}$ ), B10 (scalded at $80^{\circ} \mathrm{C}$ for $10 \mathrm{~min}$ ), $\mathrm{C} 5$ (scalded at $90{ }^{\circ} \mathrm{C} \mathrm{for} 5 \mathrm{~min}$ ) and $\mathrm{C} 10$ (scalded at $90{ }^{\circ} \mathrm{C}$ for $10 \mathrm{~min}$ ).

\section{Conclusions}

The results of this research showed that ripening time was effective on chemical, textural and color parameters of Urfa cheese. Scalding parameters on textural properties (except springiness and fracturability) of Urfa cheese were found to be important at the beginning of maturation. However, scalding parameters on hardness, cohesiveness, and gumminess were significant at the end of storage. Urfa cheese gained a more compact and uniform structure with the scalding parameters. Increasing scalding temperature and time positively improved the textural and microstructural properties of Urfa cheese. However, the effects of scalding temperature and time on the proteolysis, lipolysis, and volatiles compounds of Urfa cheese were not yet known. For this reason, further studies should be focused on the determination of the effect of scalding temperature and time on biochemical properties of lipolysis and proteolysis in Urfa cheese.

\section{Acknowledgements}

This study was supported financially by Harran University Scientific Research Projects Authority (HUBAK Project No.: 13041).

\section{References}

Akalın A S \& Karaman A D (2010). Influence of packaging conditions on the textural and sensory characteristics, microstructure and color of industrially produced Turkish white cheese during ripening. Journal of Texture Studies 41(4): 549-562 https://doi.org/10.1111/j.17454603.2010.00241.x

Alinovi M, Cordioli M, Francolino S, Locci F, Ghiglietti R, Monti L, Tidona F, Mucchetti G \& Giraffa G (2018). Effect of fermentationproduced camel chymosin on quality of Crescenza cheese. International Dairy Journal 84: 72-78 https://doi.org/10.1016/j.idairyj.2018.04.001

Aminifar M, Hamedi M, Emam-Djomeh Z \& Mehdinia A (2010). Microstructural, compositional and textural properties during ripening of Lighvan cheese, a traditional raw sheep cheese. Journal of Texture Studies 41(4): 579-593 https://doi.org/10.1111/j.17454603.2010.00244.x

Aminifar M \& Emam-Djomeh Z (2014). Changes of Texture, Microstructure and free fatty acid contents of Lighvan cheese during accelerated ripening with lipase. Journal of Agricultural Science and Technology 16: 113-123

Atasoy A F \& Türkoğlu H (2008). Changes of composition and free fatty acid contents of Urfa cheeses (a white-brined Turkish cheese) during ripening: Effects of heat treatments and starter cultures. Food Chemistry 110(3): 598-604 https://doi.org/10.1016/j.foodchem.2008.02.046

Atasoy A F, Yetişmeyen A, Türkoğlu H \& Özer B (2008). Effects of heat treatment and starter culture on the properties of traditional Urfa cheeses (a white-brined Turkish cheese) produced from bovine milk. Food Control 19(3): 278-285 https://doi.org/10.1016/j.foodcont.2007.04.004 
Atasoy A F, Hayaloglu A A, Kırmacı H, Levent O \& Türkoğlu H (2013). Effects of partial substitution of caprine for ovine milk on the volatile compounds of fresh and mature Urfa cheeses. Small Ruminant Research 115(1-3): 113-123 https://doi.org/10.1016/j.smallrumres.2013.09.002

Awad S, Hassan A N \& Muthukumarappan K (2005). Application of exopolysaccharide-producing cultures in reduced-fat cheddar cheese: Texture and melting properties. Journal of Dairy Science 88(12): 4204-4213 https://doi.org/10.3168/jds.s0022-0302(05)73106-4

Buffa M N, Trujillo A J, Pavia M \& Guamis B (2001). Changes in textural, microstructural, and colour characteristics during ripening of cheeses made from raw, pasteurized or high-pressure-treated goats' milk. International Dairy Journal 11(11): 927-934 https://doi.org/10.1016/s0958-6946(01)00141-8

El Bakry M \& Sheehan J (2014). Analysing cheese microstructure: A review of recent developments. Journal of Food Engineering 125: 8496 https://doi.org/10.1016/j.jfoodeng.2013.10.030

Eroglu A, Toker O S \& Dogan M (2016). Changes in the texture, physicochemical properties and volatile compound profiles of fresh Kashar cheese (<90 days) during ripening. International Journal of Dairy Technology 69(2): 243-253 https://doi.org/10.1111/1471-0307.12250

Famenin B K, Hosseini H, Zayeri F, Ghanati K, Lorenzo J M, Barba F J \& Mousavi Khaneghah A (2019). Effect of mechanically deboning of chicken on the rheological and sensory properties of chicken sausages. Journal of Food Processing and Preservation 43(5): e13938 https://doi.org/10.1111/jfpp.13938

Grappin R \& Beuvier E (1997). Possible Implications of Milk Pasteurization on the Manufacture and Sensory Quality of Ripened Cheese. International Dairy Journal 7(12): 751-761 https://doi.org/10.1016/s0958-6946(98)00006-5

Hayaloglu A A, Deegan K C \& Mcsweeney P L (2010). Effect of milk pasteurization and curd scalding temperature on proteolysis in Malatya, a Halloumi-type cheese. Dairy Science \& Technology 90(1): 99-109 https://doi.org/10.1051/dst/2009052

Hayaloglu A A, Karatekin B \& Gurkan H (2014). Thermal stability of chymosin or microbial coagulant in the manufacture of Malatya, a Halloumi type cheese: Proteolysis, microstructure and functional properties. International Dairy Journal 38(2): 136-144 https://doi.org/10.1016/j.idairyj.2014.04.001

Hort J \& Le Grys G (2001). Developments in the textural and rheological properties of UK Cheddar cheese during ripening. International Dairy Journal 11(4-7): 475-481 https://doi.org/10.1016/s0958-6946(01)00074-7

IDF (1993). Milk Determination of Nitrogen Content. IDF Standard 20B, International Dairy Federation, Brussels

Kahyaoglu T \& Kaya S (2003). Effects of heat treatment and fat reduction on the rheological and functional properties of Gaziantep cheese. International Dairy Journal 13(11): 867-75 https://doi.org/10.1016/s0958-6946(03)00113-4

Kahyaoglu T, Kaya S \& Kaya A (2005). Effects of fat reduction and curd dipping temperature on viscoelasticity, texture and appearance of Gaziantep cheese. Food Science and Technology International 11(3): 191-198 https://doi.org/10.1177/1082013205055002

Kırmacı H A, Hayaloğlu A A, Özer H B \& Türkoğlu H (2014). Evolution of proteolysis in Urfa cheese made from ewe's milk by wild type starter culture systems. Small Ruminant Research 119(1-3): 120-129 https://doi.org/10.1016/j.smallrumres.2014.01.006

Kirmac1 H A (2016). Effect of Wild Strains Used as Starter Cultures on Free Fatty Acid Profile of Urfa Cheese. Polish Journal of Food and Nutrition Sciences 66(4): 303-310 https://doi.org/10.1515/pjfns-2015-0034

Lawrence R C, Creamer L K \& Gilles J (1987). Texture development during cheese ripening. Journal of Dairy Science 70(8): 1748-1760 https://doi.org/10.3168/jds.s0022-0302(87)80207-2

Madadlou A, Khosroshahi A, Mousavi S M \& Djome Z E (2006). Microstructure and rheological properties of Iranian white cheese coagulated at various temperatures. Journal of Dairy Science 89(7): 2359-2364 https://doi.org/10.3168/jds.s0022-0302(06)72308-6

Motevalizadeh E, Mortazavi S A, Milani E \& Hooshmand-Dalir M A R (2018). Optimization of physicochemical and textural properties of pizza cheese fortified with soybean oil and carrot extract. Food Science \& Nutrition 6(2): 356-372 https://doi.org/10.1002/fsn3.563

Nuñez M, Guillén A M, Rodríguez-Marín M A, Marcilla A M, Gaya P \& Medina M (1991). Accelerated ripening of ewes' milk Manchego cheese: The effect of neutral proteinases. Journal of Dairy Science 74(12): 4108-4118 https://doi.org/10.3168/jds.s0022-0302(91)78605-0

Ozer H B, Uraz G, Beyzi-Yilmaz E \& Atasoy A F (2004). The effects of brine concentration and scalding on survival of some pathogens in Urfa cheese: a traditional white-brined Turkish cheese. International Journal of Food Science \& Technology 39(7): 727-735 https://doi.org/10.1111/j.1365-2621.2004.00837.x

Özer B H, Robinson R K \& Grandison A S (2003). Textural and microstructural properties of Urfa cheese (a white-brined Turkish cheese). International Journal of Dairy Technology 56(3): 171-176 https://doi.org/10.1046/j.1471-0307.2003.00100.x

Prasad N \& Alvarez V B (1999). Effect of salt and chymosin on the physico-chemical properties of Feta cheese during ripening. Journal of Dairy Science 82(6): 1061-1067 https://doi.org/10.3168/jds.s0022-0302(99)75327-0

Sahan N, Yasar K, Hayaloglu A A, Karaca O B \& Kaya A (2008). Influence of fat replacers on chemical composition, proteolysis, texture profiles, meltability and sensory properties of low-fat Kashar cheese. Journal of Dairy Research 75: 1-7 https://doi.org/10.1017/s0022029907002786

Sahingil D, Hayaloglu A A, Simsek O \& Ozer B (2014). Changes in volatile composition, proteolysis and textural and sensory properties of white-brined cheese: effects of ripening temperature and adjunct culture. Dairy Science \& Technology 94(6): 603-623 https://doi.org/10.1007/s13594-014-0185-2

Tomaszewska J, Cais-Socolinska D, Bierzunska P, Kaczynski L K, Walkowiak W \& Baranowska H M (2019). Behaviour of water in different types of goats' cheese. International Dairy Journal 95: 18-24 https://doi.org/10.1016/j.idairyj.2019.02.015

Tunick M H, Malin E L, Smith P W, Shieh J J, Sullivan B C, Mackey K L \& Holsinger V H (1993). Proteolysis and rheology of low fat and full fat Mozzarella cheeses prepared from homogenized milk. Journal of Dairy Science 76(12): 3621-3628 https://doi.org/10.3168/jds.s0022-0302(93)77703-6

Turkish Standards (1978). Determination of Fat in Cheese. TS 3046, Turkish Standards Institute, Ankara, Turkey

Turkish Standards (1989). White Cheese Standard. TS 591, Turkish Standards Institute, Ankara, Turkey 